\title{
JCAT: COLLABORATIVE ACTIVE TEXTBOOKS USING JAVA
}

\author{
Marc H. Brown \\ DEC Systems Research Center \\ 130 Lytton Ave. \\ Palo Alto, CA 94301 USA \\ Tel.: +1 4158532152 \\ mhb@pa.dec.com \\ http://www.research.digital.com/SRC/staff/mhb/bio.html \\ Roope Raisamo \\ Department of Computer Science \\ University of Tampere \\ P.O. Box 607 (Kehruukoulunkatu 1) \\ FIN-33101 Tampere, Finland \\ Tel.: +358 32157056 \\ rr@cs.uta.fi \\ http://www.uta.fi/ csrora
}

\begin{abstract}
This paper describes JCAT, a Java-based system for building Web-based collaborative active textbooks on algorithms. JCAT augments the expressive power of Web pages for publishing passive multimedia information with a full-fledged interactive algorithm animation system. The system is built in such a way that views of a running program may reside on different machines, thereby making JCAT particularly well-suited for electronic classrooms. In such a setting, an instructor controls the animation, while students view the animation by pointing their Web browsers at the appropriate page.
\end{abstract}

Keywords: Algorithm animation, program visualization, BALSA, Java, electronic textbooks, electronic classrooms, applets, remote objects 


\section{INTRODUCTION}

This paper describes JCAT, a system for building Web-based collaborative active textbooks on algorithms.

Pages of a JCAT textbook consist of passive multimedia material combined with interactive algorithm animations. The passive multimedia is specified using HTML and can exploit the expressive power of Web pages (e.g., text, audio, and video). The animations are prepared using a full-fledged algorithm animation system.

From the user's point of view, there are multiple views of a running program, and all are updated simultaneously as the program runs. In addition, there are control panels for starting, pausing, and stopping the animation, for adjusting its speed, and for giving input data to the algorithm.

Each view in JCAT, as well as the control panels, is implemented as an applet. Because JCAT is based on Java's Remote Method Invocation (RMI) technology for allowing applets on different machines to communicate with each other [Sun96], the views of an algorithm can reside on any machine. Thus, in an electronic classroom, an instructor can control an animation on his machine (specifying the input, single-stepping the program to some point, and so on), and students in the class can see views of the program on their machines by pointing their browsers at the appropriate page.

The screen image at the top of the next column shows Netscape Navigator displaying a page from a prototype JCAT textbook on binpacking algorithms. Although the page is not very glamorous (there is no audio, there is no video, there is little explanatory text), it does show the basics of a page from a JCAT textbook. We'll look at this screen image in more detail later.

JCAT is a Java implementation of the CAT system developed by Brown and Najork [Brown-Najork96]. CAT was implemented using a family of home-built Web browsers that supported applets written in Obliq [Cardelli95], an inherently distributed language. Although

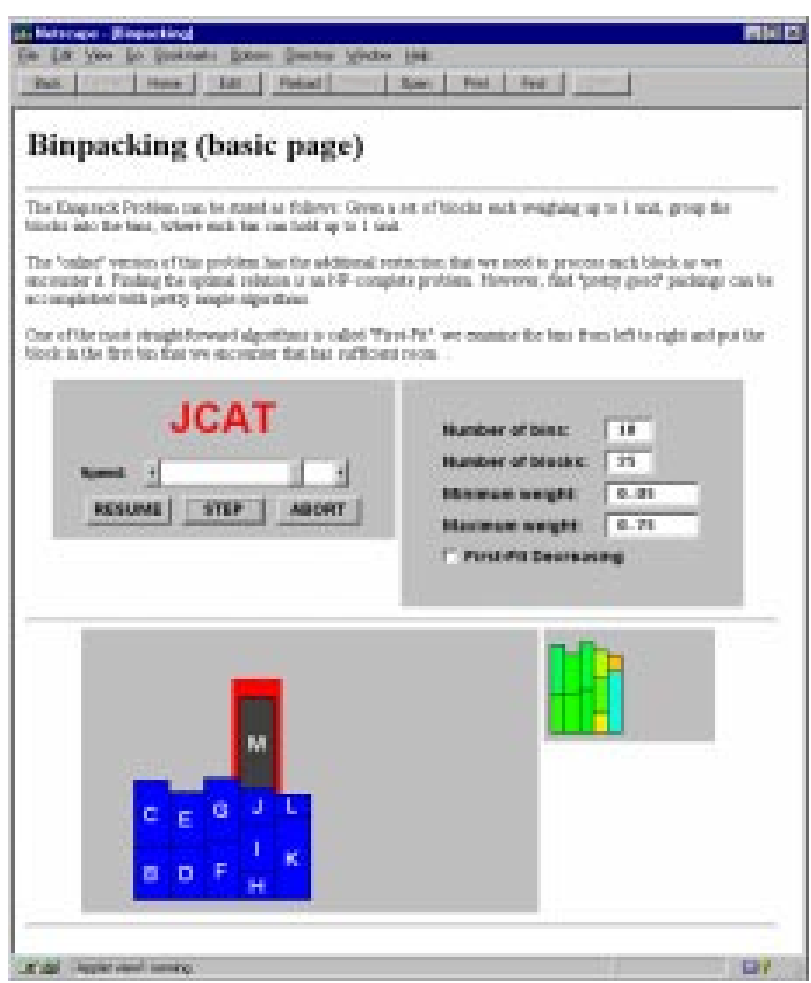

Java does not have the same level of support that Obliq has for applets on different machines to communicate with one another, the RMI library provides much of the same functionality.

There are numerous Java applets of animated algorithms available on the Web. JCAT (and CAT) are unique in a number of ways. To the user, J/CAT animations are the only Web-based algorithm animations with multiple views of a program. In addition, J/CAT animations are the only Web-based algorithm animations with support for collaboration. To the programmer implementing an algorithm animation, J/CAT is the only system with support for constructing algorithm animations. The support comes in two flavors: there's a bona fide framework for separating the algorithm from the views, and there's a rich animation package. Both the framework [Brown-Sedgewick84] and the animation package [DeTreville93] are wellproven in practice.

The remainder of this paper examines JCAT from three perspectives: the end user, the programmer, and the system implementor. 


\section{THE USER'S VIEW}

We envision the JCAT system being used as both a stand-alone electronic textbook on algorithms and a collaborative active textbook in an electronic classroom.

The screen image on the previous page shows a page from a prototype stand-alone electronic textbook being developed with JCAT. The pages are from a chapter on binpacking. The binpacking problem is as follows: Given a set of blocks each weighing up to 1 unit, group the blocks into the fewest bins possible, where each bin can hold up to 1 unit. The "online" version of this problem has the additional restriction that each block must be processed as it is encountered in the input stream. Although the optimal solution to this problem is NPcomplete, a "pretty good" packing can be accomplished with the following simple algorithm: Examine the bins in order and put the block in the first bin encountered that has sufficient room. This algorithm is called first-fit binpacking.

The top-left applet is the JCAT control panel. It allows the user to start and stop the algorithm, advance the algorithm step-by-step, and adjust the speed of the animation. The JCAT control panel is algorithm-independent; this applet is used to control all algorithms in the JCAT system.

The applet at the top-right is an algorithm input dialog that is used for specifying input to the algorithm. This applet is specific to each algorithm. The algorithm input dialog used for binpacking algorithms allows users to specify the number of bins available for packing, the number of blocks to pack, the possible weights of each block, and whether to sort all of the blocks into decreasing order before trying to pack them.

The two applets below the horizontal line are views. The large applet on the left is the Probing view; it shows each block as a vertical bar whose height reflects the weight of the block. As the algorithm examines the bins, the new block is graphically shown on the bin being examined. Once a bin is found with enough room for the new block, the color of the new block changes from gray to blue. The smaller applet on the right is the Packing view; it shows how the blocks have been arranged into the bins. Color is used to redundantly encode the weight of each block.

The figures at the bottom of this page show the value of using color in the Packing view. In both screen images, the algorithm was run with the same set of 128 blocks uniformly distributed in the range of 0.0 to 0.5 . In the image at the right, the blocks were first sorted into decreasing order (an option on the algorithm input dialog). The pictures don't lie: the packing is more dense when the blocks are first sorted into decreasing order. In fact, the intuition behind the formal analysis of binpacking algorithms was developed by Jon Bentley and his colleagues with the aid of an algorithm animation he had done [Bentley91], quite similar to the images below!

The screen images on the next page show how JCAT can be used as a collaborative active textbook in an electronic classroom. Again, we use the domain of binpacking.

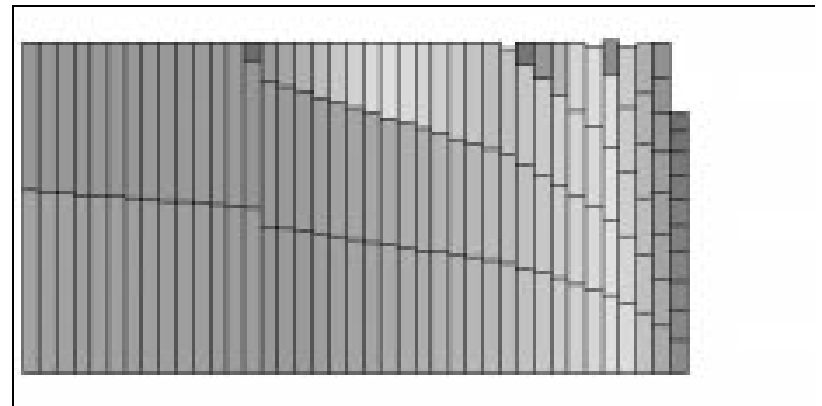


The screen image at the right shows the Web page that an instructor is visiting in his browser. It contains the JCAT control panel, the algorithm input dialog, and the Probing view. We saw these three applets before. The instructor can control the algorithm as before.

The screen images below show the screens of two students at the same time that the screen image of the instructor's PC was captured. The students are looking at different pages; both of the pages contain views that are displaying the algorithm that the instructor is controlling.

The student at the left is using Netscape Navigator; the page he is visiting contains two views: the Glossary view at the top and the Probing view at the bottom.

The student at the right is using Microsoft Internet Explorer. The page he is visiting contains three views: The Packing view and the Probing view on the top, and the Glossary view at the bottom.

Note how the Probing view and the Packing view scale their contents to fit the page real estate allocated to them. When the screen images were captured, there were eight active applets from the JCAT system point of view, three on the instructor's computer, two on one student's computer and three on the other student's computer.

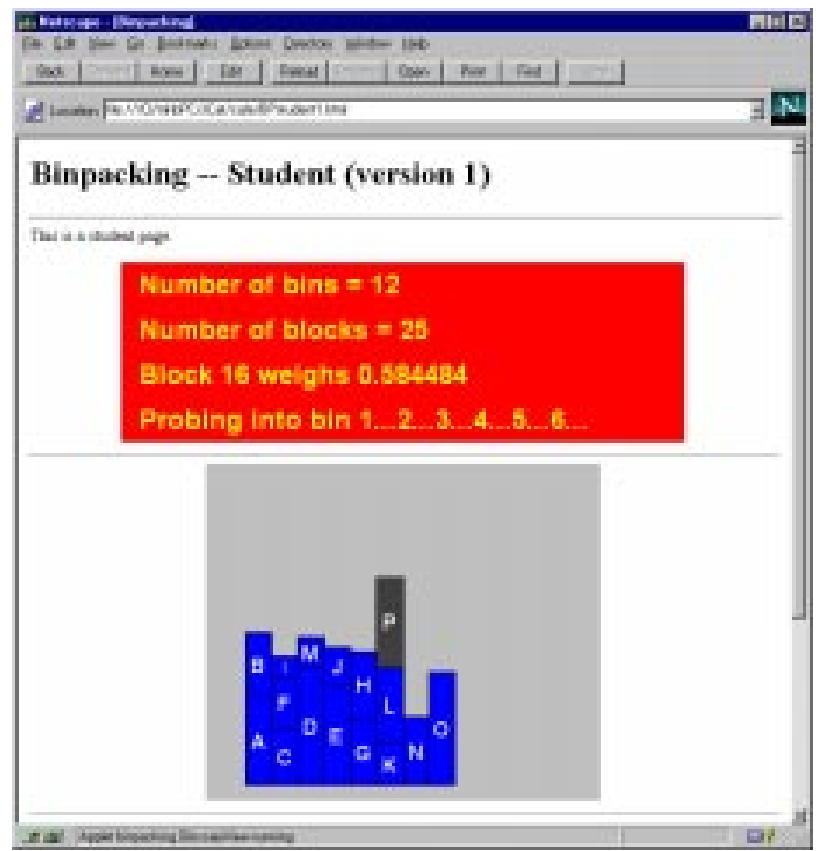

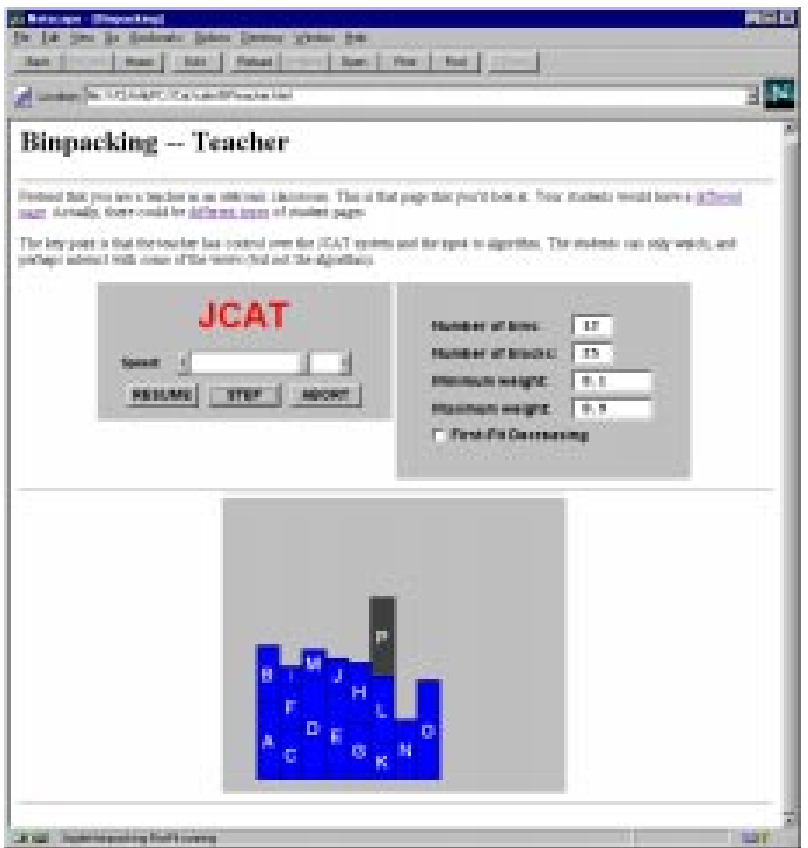

\section{THE PROGRAMMER'S VIEW}

The framework for animating algorithms follows the model pioneered by BALSA [Brown-Sedgewick84]: Strategically important points of an algorithm are annotated with procedure calls that generate interesting events (e.g., "swap elements" in a sorting algorithm). These events are reported to an event manager, which in turn forwards them to all registered views. Each view responds to each interesting event by drawing appropriate images.

The task of preparing a page of a JCAT textbook consists of four parts: defining the interesting events for the algorithm;

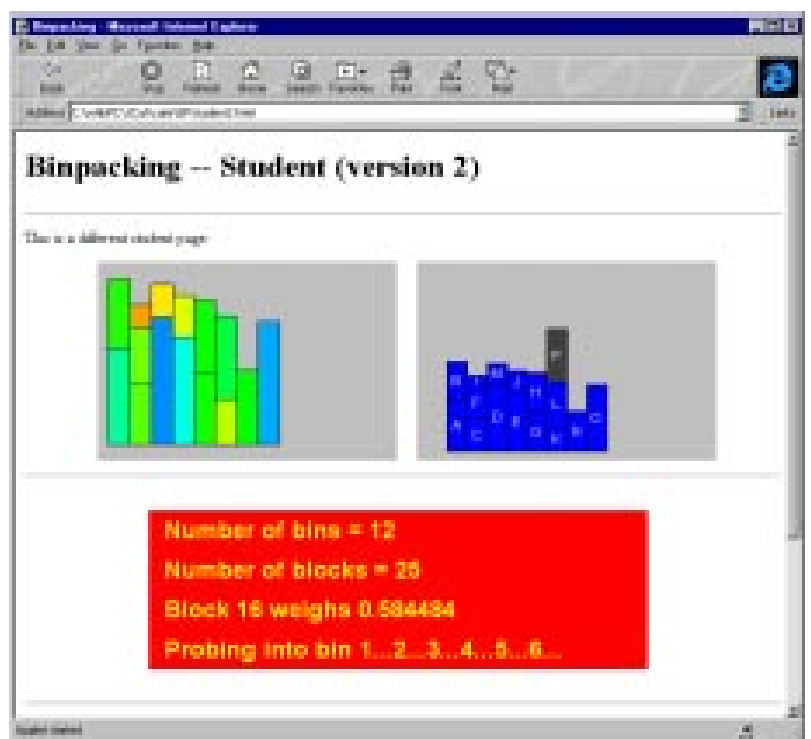


implementing the algorithm and annotating it with the events; implementing one or more views; and finally, creating Web pages that make use of the algorithm and views. The Web pages are prepared using HTML; the events, algorithm, and views are implemented in Java.

The rest of the section shows how the binpacking animations seen before were implemented.

\section{The Interesting Events}

In JCAT, interesting events are specified as a Java interface. Here are the interesting events for binpacking algorithms:

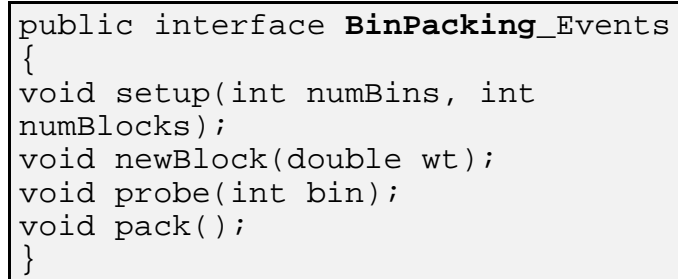

The setup event is called once when the algorithm starts, to communicate to the views how many blocks will be processed and the maximum number of bins available. The newBlock event is called each time the algorithm encounters a new block, whose weight is specified as the parameter. The probe event is called each time the algorithm checks if the new block can be packed into the bin specified as a parameter. The pack event is called to signal that the last bin probed is where the new block will be placed.

It is important to realize that there is no right or wrong set of events or parameters to them. Another programmer implementing binpacking algorithms might have chosen a different set of events or different parameters. The choice of events and parameters will affect how much additional state each view must maintain, since views do not have access to the algorithm's data structures.

The name of the interface is special: as we shall see, JCAT will use this interface to create some additional objects that are needed for the algorithm and views to communicate. We have typeset Binpacking in bold here, and in the places in the example algorithm and view where auxiliary objects have been created.

\section{The Algorithm}

An algorithm is a subclass of the JCAT class AlgorithmApplet, which in turn is a subclass of the standard Java Applet class. More specifically, an algorithm is a subclass of the type of algorithm for which it can generate interesting events, and this class is a subclass of AlgorithmApplet. For example, the firstfit binpacking algorithm class is a subclass of BinpackingAlgorithm, which indicates that it's an algorithm that can generate the events defined in the Binpacking_Events interface. And BinpackingAlgorithm is a subclass of AlgorithmApplet, which is a subclass of the standard Java Applet class.

The actual algorithm appears in the method called algorithm. The following code shows the first-fit binpacking. The event annotations are shown italics.

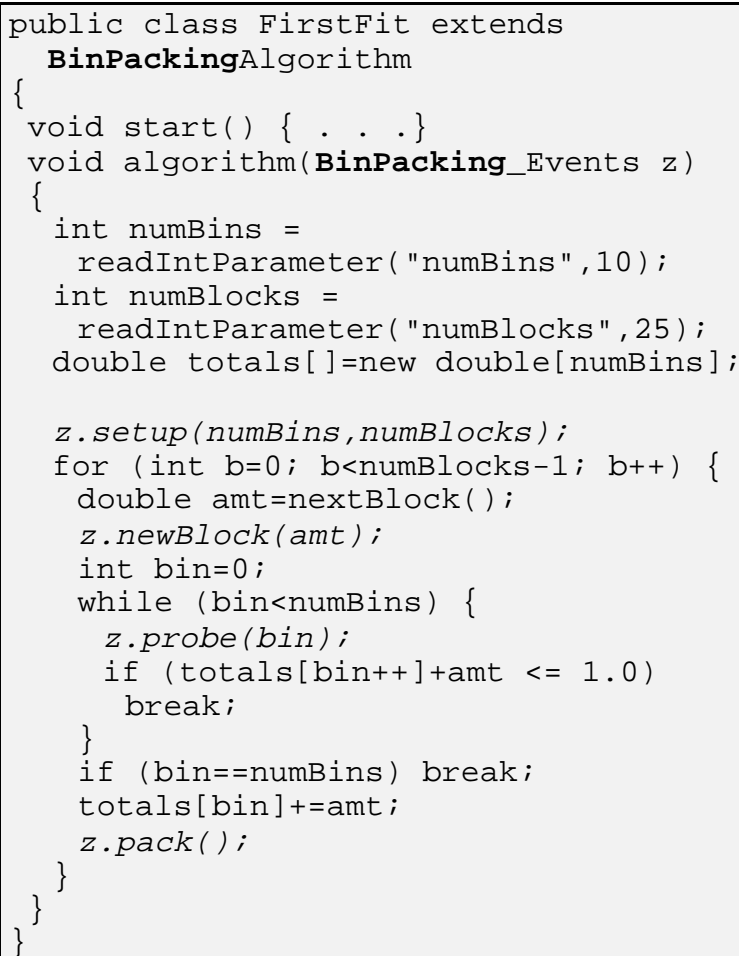

The algorithm method makes use of internal procedures readintParameter and nextBlock (not shown) for retrieving the input data specified by the user. 
This algorithm's start method (also not shown) initializes the graphical user interface of the algorithm input dialog.

\section{A View}

A view in JCAT is an applet with an additional set of methods corresponding to each interesting event defined in the events interface. For example, binpacking views are subclasses of BinpackingView, and this class is a subclass of the JCAT class ViewApplet that also implements the Binpacking_Events interface. A ViewApplet is a subclass of the standard Java Applet class.

The code at the right is the actual code for the Probing view shown in the previous screen images.

As mentioned, views of an algorithm implement the methods that are defined in the interesting event interface. The body of each method is responsible for updating the screen in a way that is meaningful for the view. For example, the probe method smoothly slides the rectangle representing the block being processed from its current position to a position on the bin being probed, specified as a parameter to the event. In addition, it records which bin is being probed so the pack event can update an array that maintains the total weight of the blocks in each bin.

The class GP (not shown) is a rich, highlevel animation package based on the metaphor of a graph consisting of vertices and edges [DeTreville93]. Each vertex has various attributes associated, such as position, size, shape, color, border width, and label. An edge connects two vertices and has attributes such as color and thickness. Vertices can be repositioned, and such movement can be shown by smooth animation, inspired by the TANGO system [Stasko90].

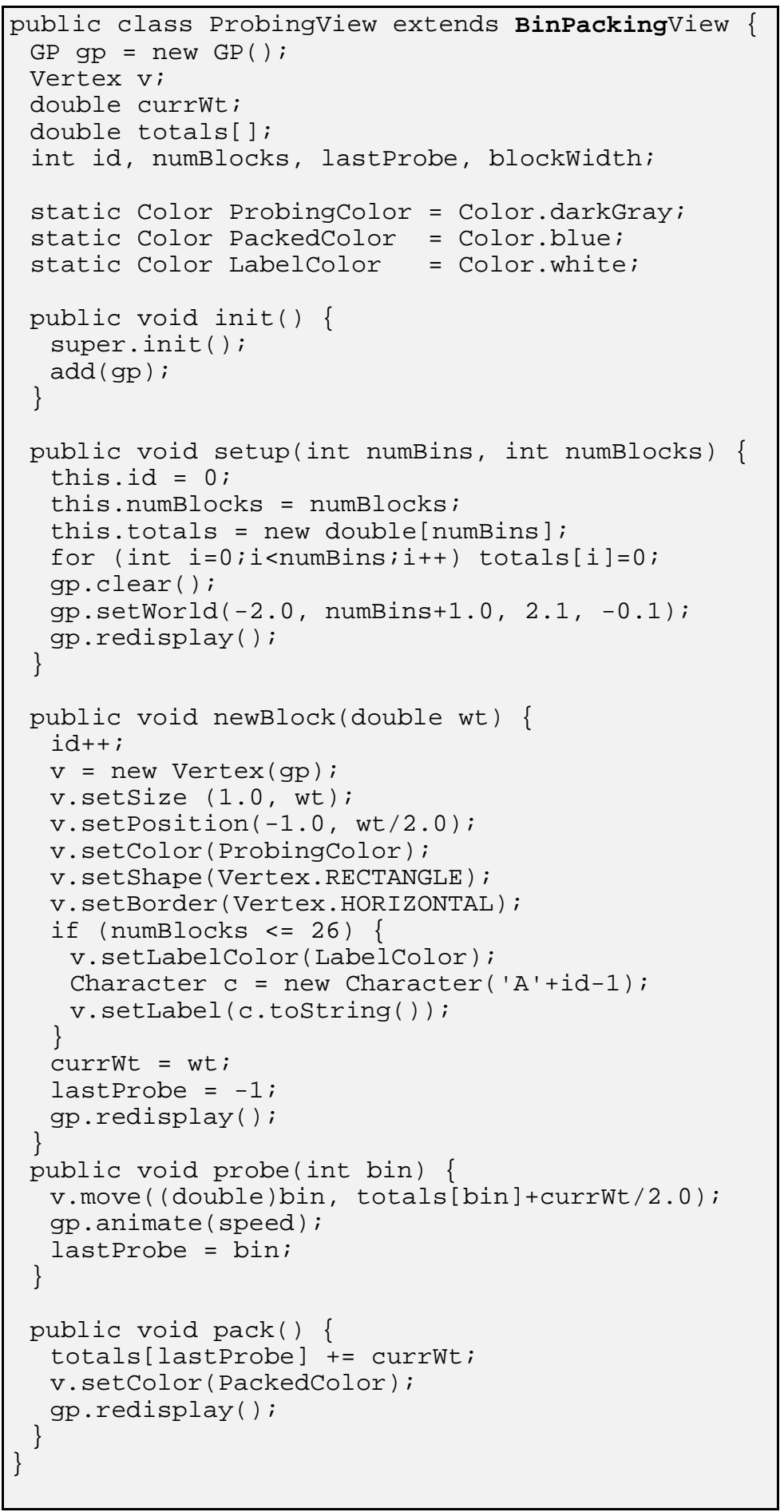




\section{SYSTEM IMPLEMENTATION}

As mentioned before, the views, algorithm, and JCAT control panel are all Java applets. They can be added to HTML pages in the standard way by using the APPLET tag.

The complete JCAT system consists of the applets, an event dispatcher application, and a class generator application. The event dispatcher application is run on the server where the active textbook pages are loaded from. The class generator application is used by programmers to generate the algorithm-specific superclasses, for example the BinPackingAlgorithm and BinPackingView in the previous section.

\section{Class structure}

The figure below shows the class structure of JCAT, using the binpacking example from before. The classes written by the user were described in the previous section.

The system generates (from the interface containing the interesting events) classes BinPacking_z, BinPackingView and BinPackingAlgorithm. These classes are compiled with the actual view and algorithm classes and take care of managing all the RMI connections that are not visible for the programmer.

The classes EventSender, ViewApplet, AlgorithmApplet and ControlApplet are all part of the JCAT system; these do not change when JCAT is used to animate algorithms from other domains. However, when the programmer writes new algorithms or views, these system classes are subclassed. Of the JCAT system classes, only the ControlApplet class is used to create final objects.

The only automatically generated class that has not been described is BinPacking_z. It is essential in the event passing procedure, which we shall describe next.

\section{Event passing}

The problem in passing the interesting events between the applets in the system is that applets can communicate only with the server where they came from. In essence, applets can't communicate with each other if they are situated in many different machines. To solve this problem we run the event dispatcher as a Java application in the Web server which has the HTML pages and Java classes for the algorithm. The event dispatcher forwards an event from the algorithm to all its views that

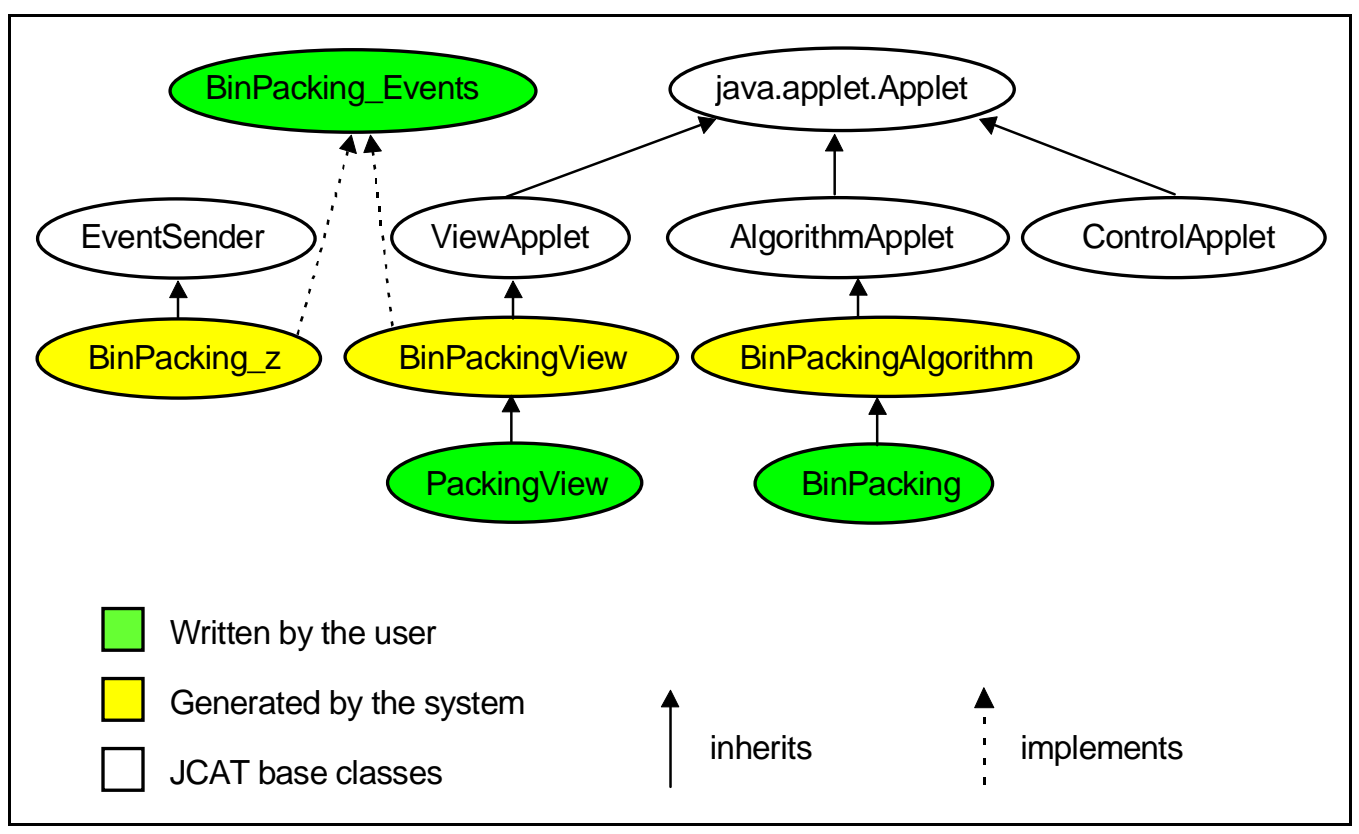


can reside in different machines. This is possible because Java applications do not need to follow the same network restrictions as applets. The figure below shows the way the events are sent in the system by showing the method calls that are made between the algorithm applet and its views.

In our example, the interesting events are called in the BinPacking algorithm through an instance of the class BinPacking_z, a subclass of EventSender. When an interesting event is called in this object, it packs the event in a general Event object, which is then conveyed to the event dispatcher. This way we can keep the event dispatching system unchanged when different algorithms use different interesting events. Otherwise any new event would require a new method added in every class that lies between the algorithm and its views in the example.

When the event dispatcher receives a new event object, it sends it to every view of this algorithm. Internally it is using separate threads for receiving and sending the events.

Finally, when the event dispatcher calls a view applet to send the Event object to it, the view applet calls a method of this object, which in turn calls the appropriate interesting event method in the current view. The view can handle this last method call any way it likes to or it can be left unprocessed by implementing an empty method for any event.

\section{The use of Java RMI package}

The primary reason for using the Java RMI package was to exploit its high-level functionality in communicating between Java programs in different machines. This saves us from then burden of having to serialize and unpack the data on a socket by ourselves. When the RMI system is in use, the syntax of calling a method in a remote machine is similar to the syntax of calling a local method.

The use of the package is not visible for the programmer. Everything that is needed is handled by the superclasses ViewApplet and AlgorithmApplet. When a Java applet is started, it first creates an AnimConnection remote object that is used to call methods in the event dispatcher object. Then it calls an event dispatcher method to create a client object that is used to call methods in the applet from the dispatcher.

Java RMI works by creating skeleton and stub classes for every class that is used to create remote objects. The actual data transfer happens between these classes, but they are not used directly. Remote objects have to be used through remote interfaces, which are normal

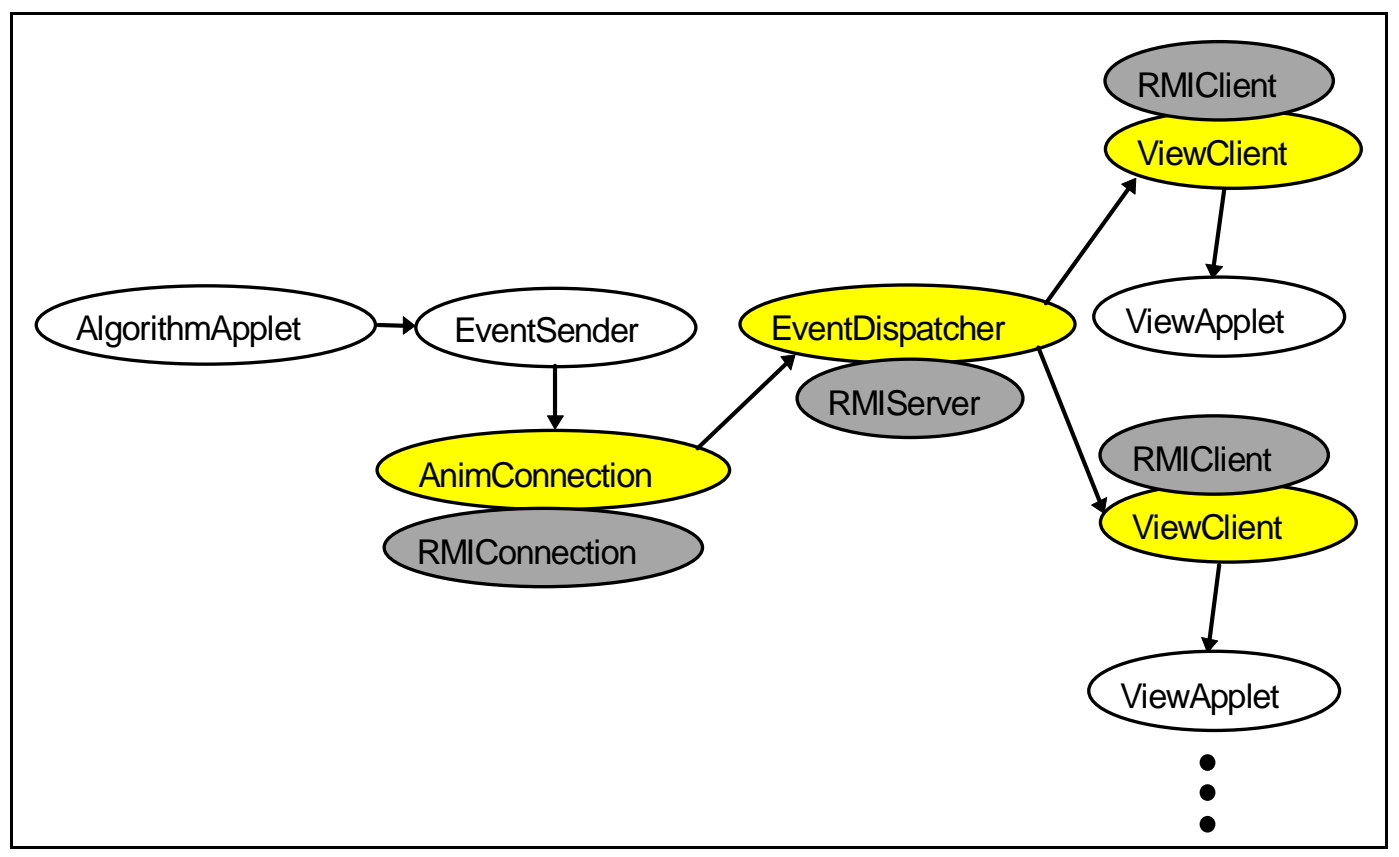


Java interfaces extending the RMI interface Remote. In the event dispatching figure there are three of this kind of interfaces, RMIConnection, RMIServer and RMIClient. Classes AnimConnection, EventDispatcher and ViewClient implement these interfaces and extend an RMI class UnicastRemoteServer to be able to answer to remote calls.

\section{CONCLUSION}

This paper has described the JCAT system, a Java-based collaborative active textbook for algorithms. JCAT augments the expressive power of HTML (which is passive) with interactive animations of the algorithms in a distributed environment.

\section{ACKNOWLEDGMENTS}

Marc Najork developed CAT along with the first author. The system described in this paper borrows heavily from the CAT system.

\section{REFERENCES}

Bentley, J.L., Kernighan, B.W. 1991 A System for Algorithm Animation. Computing Systems, 4(1), 5-30, Winter 1991.
Brown, M.H., Sedgewick, R. 1984 A System for Algorithm Animation, Computer Graphics, 18(3), 177-186, July 1984.

Brown, M.H., Najork, M.A. 1996 Collaborative Active Textbooks: A WebBased Algorithm Animation System for an Electronic Classroom. Proceedings 1996 IEEE Symposium on Visual Languages, 266-275.

Cardelli, L. 1995 A Language with Distributed Scope. Computing Systems, 8(1), 27-59, January 1995.

DeTreville, J.D. 1993 The GraphVBT Interface for Programming Algorithm Animations. Proceedings 1993 IEEE Symposium on Visual Languages, 26-31.

Sun Microsystems, Inc. 1996 Java(TM) Remote Method Invocation Specification. http: //chatsubo. javasoft.com/ current/doc/rmi-spec/rmiTOC.doc.html

Stasko, J.T. 1990 TANGO: A Framework and System for Algorithm Animation. IEEE Computer, 23(9):27-39, September 1990. 